\title{
Mioclonía palatina esencial. Reporte de un caso pediátrico
} Essential palatal myoclonus. A pediatric case report

\author{
Dra. Roxana Spini ${ }^{a}$ Dra. Daniela Cohen ${ }^{a}$ Dra. Marian Juchli ${ }^{a}$ y Dra. Lilian Ibarra ${ }^{(+) b}$
}

\begin{abstract}
RESUMEN
La mioclonía palatina esencial es una entidad otoneurológica rara. Se caracteriza por movimientos involuntarios de los músculos del paladar blando que causan un tinnitus objetivo. La mioclonía del paladar se clasifica en dos tipos: secundaria y primaria (mioclonía palatina esencial); esta última es más frecuente en pediatría. La tomografía computada y la resonancia magnética de cerebro normal orientan al diagnóstico. La mioclonía palatina esencial, generalmente, se resuelve en forma espontánea.

Se presenta a una paciente de 8 años deedad con un "clic" rápido en forma rítmica en su boca que cedía en forma espontánea. Palabras clave: mioclonía palatina, mioclonía esencial, acúfeno, pediatría.
\end{abstract}

\begin{abstract}
Essential palatal myoclonus is a rare neurological disorder characterized by involuntary movements of the soft palate musculature causing objective-clicking tinnitus. The palatal myoclonus is classified in two forms, secondary and essential palatal myoclonus. Primary (essential) palatal myoclonus is the most common type in childhood. Normal computed tomography and magnetic resonance guide the diagnosis. Spontaneous resolution usually occurs in the essential type of palatal myoclonus.

In this report, we present an 8-year-old child making rhythmic, rapid clicking noises from her throat with spontaneous resolution.
\end{abstract}

Key words: palatal myoclonus, essential tremor, tinnitus, pediatrics.

http: / / dx.doi.org/10.5546/ aap.2021.e54

Cómo citar: Spini R, Cohen D, Juchli M, Ibarra L. Mioclonía palatina esencial. Reporte de un caso pediátrico. Arch Argent Pediatr 2021;119(1):e54-e57.

a. División de Otorrinolaringología.

b. División de Neurología.

Hospital General de Niños Pedro de Elizalde,

Ciudad Autónoma de Buenos Aires.

Correspondencia:

Dra. Roxana Spini: spiniroxana@intramed.net

Financiamiento: Ninguno.

Conflicto de intereses: Ninguno que declarar.

Recibido: 12-6-2020

Aceptado: 9-9-2020

\section{INTRODUCCIÓN}

La mioclonía palatina esencial (MPE) es una entidad rara. Generalmente, se acompaña con tinnitus objetivo. ${ }^{1}$ Existen muy pocos casos reportados tanto en pacientes adultos como en pacientes pediátricos.

La mioclonía palatina puede ser de origen secundario (isquemia cerebral, traumática, tumoral, infecciosa o desmielinizante) o de origen primario (idiopático o esencial). La mioclonía palatina secundaria es más frecuente que la primaria. ${ }^{1}$ La MPE es más común en la edad pediátrica. ${ }^{2,3}$ Generalmente, presenta resolución espontánea. El objetivo es presentar un caso clínico de MPE en una niña de 8 años y conocer los diagnósticos diferenciales y su tratamiento.

\section{CASO CLÍNICO}

Paciente de 8 años, de sexo femenino, previamente sana, sin antecedentes perinatológicos ni personales de importancia. Consultó al Servicio de Otorrinolaringología del Hospital de Niños Pedro de Elizalde por un "ruido en la boca" de 48 horas de evolución. La niña refirió un ruido tipo "clic" que provenía de la boca, que lo escuchaba ella y su familia. Lo caracterizaba como ruido molesto porque no cedía en ningún momento, ni siquiera en el sueño.

Se encontraba en buen estado general, afebril. Al momento del examen físico, en las fauces, se visualizaron contracciones espasmódicas del velo del paladar en forma rítmica permanentemente y bilateral (ver vídeo en: https: / / www.youtube. $\mathrm{com} /$ watch? $\mathrm{v}=\mathrm{z} 8 \mathrm{~b} 1 \mathrm{sg}$ UEwds). No se observó asimetría del paladar (Figura 1). En la otoscopia, presentó oídos ventilados. La paciente no tenía ningún otro síntoma asociado. Negaba dificultad para tragar y sialorrea. Fue evaluada por el Servicio de Foniatría, quienes descartaron alteraciones en el primero y segundo tiempo de la deglución.

Se realizó una interconsulta con Neurología y, en conjunto, se decidió su internación para estudio. Durante la evolución, se realizó una tomografía del macizo cráneo-facial, que mostró hipertrofia de la mucosa del seno maxilar izquierdo (hallazgo), y de cerebro con contraste, 
que se informó como normal (Figura 2). Se realizó una resonancia magnética de cerebro y tronco de encéfalo con gadolinio y un electroencefalograma, cuyos resultados fueron normales. Se descartó, de esta forma, la patología orgánica de cerebro y tronco de encéfalo, así como un proceso convulsivo.

Al quinto día de internación, el movimiento paroxístico del paladar cedió en forma espontánea, y se otorgó el egreso hospitalario asumiendo el cuadro como una MPE. Volvió a tener el episodio dos meses después con una duración de un día, que cedió espontáneamente. En la actualidad, se encuentra libre de episodios luego de dos años del primer evento.

\section{DISCUSIÓN}

El "temblor del paladar" o también llamado MPE es un trastorno neurootológico. ${ }^{1}$ Es una

FIgURA 1. Examen de fauces simétrica

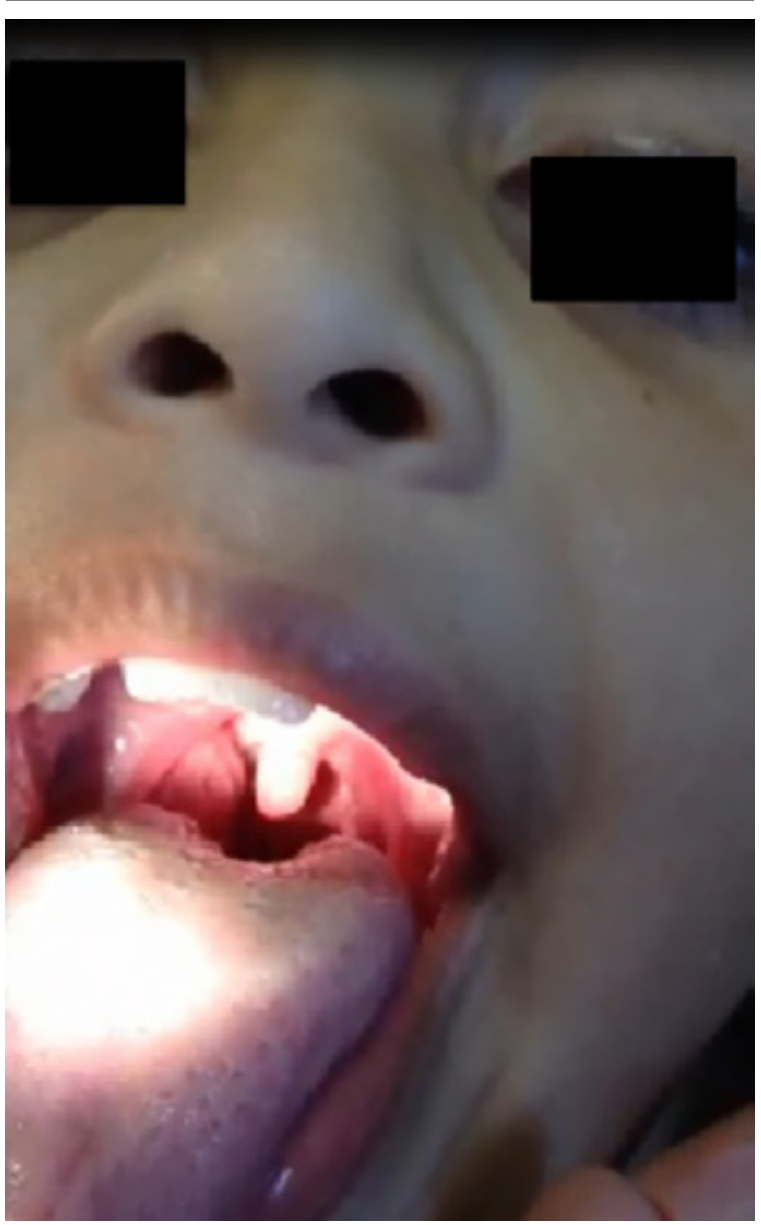

La foto fue tomada mientras las contracciones rítmicas continuaban en forma permanente. rara alteración del movimiento segmentario caracterizada por espasmos rítmicos simétricos de los músculos del paladar blando sin lesiones cerebelosas o del tronco encefálico. ${ }^{1,4}$

Goltz y cols., plantean que existe una activación neuronal bilateral del putamen de causa incierta. ${ }^{5}$ El músculo implicado es el tensor del velo del paladar que, al contraerse, genera la apertura forzada de la trompa de Eustaquio, que ocasiona el tinnitus. ${ }^{1}$

En esta entidad, se producen movimientos simétricos entre 100 y 150 veces por minuto, que persisten durante el sueño. El paciente refiere, clínicamente, la presencia de un "clic" en el oído, entre 1 y 2 "clics" por segundo, que se vuelve un ruido molesto y discapacitante. Este puede ser percibido por el examinador como un acúfeno o tinnitus objetivo. La causa de estos acúfenos no se conoce, pero es más frecuentemente observada en la MPE. ${ }^{6}$

Como diagnóstico diferencial, se debe tener en cuenta la mioclonía palatina secundaria, en la que el músculo implicado es el elevador del velo. Presenta participación ocasional de los músculos del ojo o de la faringe. El tinnitus está ausente. Generalmente, las contracciones musculares son continuas, involuntarias y de por vida. El diagnóstico de la MPE se realiza por descarte.

También debe tenerse en cuenta la mioclonía del oído medio, que se caracteriza por tinnitus objetivo uni- o bilateral. No hay afectación del paladar. El músculo afectado, en estos casos, es el músculo tensor del martillo o el músculo estapedial. ${ }^{7}$

La tomografía con contraste y la resonancia magnética del cerebro y el electroencefalograma son normales en la MPE. En cambio, en la forma secundaria, aparecen lesiones cerebrales por una variedad de etiologías, tales como infecciones, vasculitis, enfermedades desmielinizantes, lesión cerebral traumática o accidente cerebrovascular.

La MPE se puede subdividir en voluntaria (a veces, llamada psicógena) $)^{3}$ o involuntaria. El tratamiento de la MPE voluntaria consiste en la administración de placebo junto con psicoterapia. ${ }^{1}$ En el caso de la MPE involuntaria, se basa en la psicoprofilaxis y fármacos, como ácido valproico, carbamazepina o clonazepam. ${ }^{8}$ La inyección de toxina botulínica en la inserción del elevador y / o tensor de músculos palatinos ha sido implementada en algunos $\operatorname{casos}^{9,10}$ con resultados satisfactorios porque cedió la mioclonía del paladar, pero, en ambos casos, tuvieron consecuencias por la generación de un 
velo insuficiente mientras duró el efecto de la toxina. A pesar de esto, Wan y col., consideraron el tratamiento con toxina botulínica como segura y efectiva. ${ }^{10}$

Si bien existen varios casos reportados de MPE en pediatría, no hay estadística publicada de su frecuencia. Se considera importante realizar el diagnóstico diferencial con mioclonía secundaria y conocer el manejo multidisciplinario de ambas patologías. La MPE presenta tinnitus objetivo sin lesiones cerebrales y, generalmente, es de resolución espontánea. En cambio, la mioclonía palatina secundaria presenta lesión cerebral sin tinnitus y es persistente y de por vida.

\section{REFERENCIAS}

1. Schwartz R, Bahadori R, Myseros J. Loud Clicking Sounds Associated With Rapid Soft Palate Muscle Contractions. Pediatr Emer Care. 2012; 28(2):158-9.

2. Campistol-Plana J, Majumdar A, Fernández-Álvarez E. Palatal tremor in childhood: clinical and therapeutic considerations. Dev Med Child Neurol. 2006; 48(12):982-4.

3. Margari F, Giannella G, Lecce PA, Fanizzi P, et al. A childhood case of symptomatic essential palatal tremor. Neuropsychiatr Dis Treat. 2011; 7:223-7.

4. De Régloix SB, Marhic A, Pons Y. An Unusual Cause of Tinnitus: Essential Palatal Myoclonus. J R Army Med Corps. 2017; 163(3):e1.

5. Golz A, Fradis M, Netzer A, Ridder G, etal. Bilateral Tinnitus due to Middle-Ear Myoclonus. Int Tinnitus J. 2003; 9(1):52-5.

6. Saeed R, Mohammed Z. Essential rhythmic palatal myoclonus in a 51-year-old man. Oxf Med Case Reports. 2016; 2016(8): omw056.

FIgURA 2. Tomografía computada de cerebro
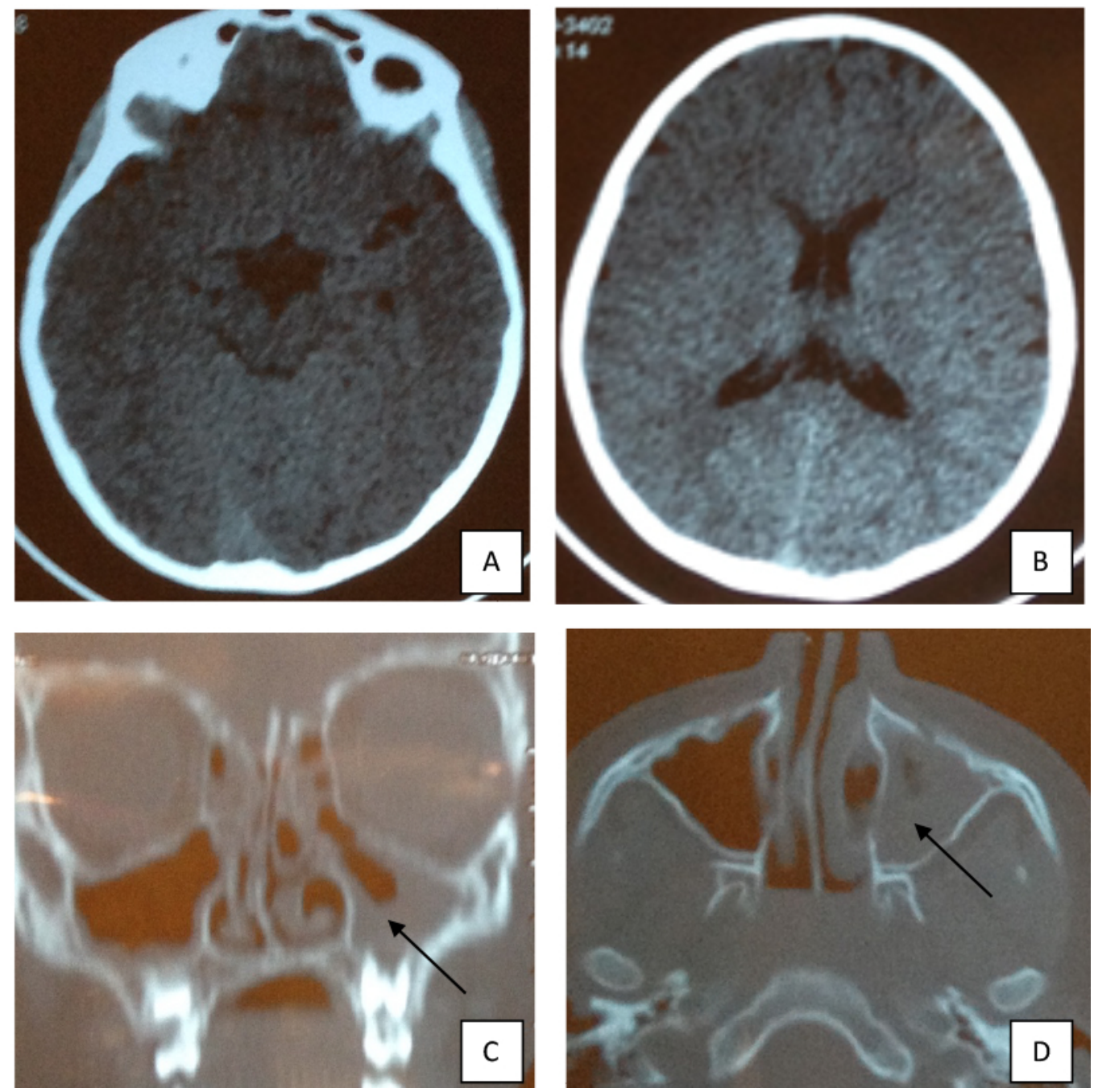

A y B. Cortes axiales: normales.

C y D. Cortes axiales del macizo cráneo-facial: hipertrofia de la mucosa del seno maxilar izquierdo (flechas). 
7. Conill Tobías N, De Paula Vernetta C, García Callejo F, et al. Mioclonía palatal como causa de acúfeno objetivo. Uso de toxina botulínica: a propósito de un caso. Acta Otorrinolaringol Esp. 2012; 63(5):391-2.

8. Wan TK, Chen J T, Wang PC. EMG-guided salpingopharyngeus Botox ${ }^{\circledR}$ injection for palatal myoclonus. B-ENT. 2013; 9(1):67-9.
9. Haller S, Winkler DT, Gobbi C, Lyrer P, et al. Prominent Activation of the Putamen during Essential Palatal Tremor: A Functional MR Imaging Case Study. AJNR Am J Neuroradiol. 2006; 27(6):1272-4.

10. Scozzafava J, Yager J. Essential Palatal Myoclonus. Images in Clinical Medicine. N Engl J Med. 2010; 362(21):e64. 